\title{
La construcción del oficio de estudiante universitario en ingresantes a la Facultad de Ciencias Químicas en dos universidades de Córdoba
}

The construction of the profession of university student in entrants to the Faculty of Chemical Sciences in two universities of Córdoba

\author{
Rosana Carina Enrico ${ }^{1}$
}

\begin{abstract}
Resumen: El presente artículo se propone socializar algunos de los hallazgos obtenidos en una investigación realizada para obtener el grado de Magíster en Investigación Educativa. Dicha investigación aborda los capitales culturales y la construcción del oficio de estudiante universitario en jóvenes migrantes. En esta oportunidad, haremos foco -particularmente- en el proceso de construcción del oficio de estudiante. Para dar cuenta de este proceso, se intentará dar respuesta a los siguientes interrogantes: ¿Qué prácticas y acciones despliega el estudiante para ejercer el oficio de estudiante universitario? Cumplir con las exigencias del nivel universitario, ¿supone realizar cambios en la forma y los recursos que utiliza para estudiar? De ser así, ¿cuáles son los cambios más significativos?

Para responder estos interrogantes, se recuperan las voces de ingresantes a la Facultad de Ciencias Químicas de la Universidad Católica de Córdoba y de la Universidad Nacional de Córdoba.
\end{abstract}

Palabras clave: universidad, estudiante, aprendizaje.

\footnotetext{
${ }^{1}$ Magíster en Investigación Educativa. Licenciada y Profesora en Psicopedagogía. Docente e investigadora de la Universidad Católica de Córdoba y de la Universidad Provincial de Córdoba, Argentina. Correo electrónico: rosanaenrico@yahoo.com.ar.
}

Diálogos Pedagágicas. ISSN en línea: 2524-9274.

Año XVII, No 34, octubre 2019 - marzo 2020. Pág. 18-36.

DOI: http://dx.doi.org/10.22529/dp.2019.17(34)02 / Recibido: 21-09-2018 / Aprobado: 3-07-2019.

cc)(-) $\odot$ Artículo publicado bajo Licencia Creative Commons Atribución-NoComercial-SinDerivar. (c) Universidad Católica de Córdoba. 


\begin{abstract}
This article has the purpose of socializing some findings from a research to achieve the Magister Degree in Educational Research. It approaches cultural capitals and the construction of the profession of university student in young immigrants. In this opportunity, we will particularly focus on the process of construction of the student profession. In order to account for this process, we will try to answer the following questions: What practices and actions does the student deploy to be a university student? Complying with the requirements at university level, does it imply changes on the manner and the resources used to study? If so, what are the most significant changes?

In order to answer these questions, we will resort to the opinions of the freshmen of the Faculty of Chemical Sciences from the Catholic University de Córdoba and the National University Córdoba.
\end{abstract}

Keywords: university, student, learning.

\title{
Introducción
}

El ingreso a la vida universitaria es una etapa de transición en la que el estudiante se enfrenta a una situación totalmente novedosa que le demanda realizar cambios personales significativos para integrarse al nuevo contexto académico y social. Las disposiciones y recursos interiorizados en su trayectoria de vida se constituyen en el soporte a partir del cual interactúa con el nuevo contexto, a la vez que se modifican y enriquecen con la incorporación de nuevas experiencias sociales y educativas. Al respecto, Coulon (1995) plantea que la primera tarea con la que se encuentra el estudiante en su ingreso a la universidad es aprender el "oficio de estudiante". Lo define como una transición de alumno (estudiante de nivel secundario) a estudiante (de nivel superior), en un proceso de afiliación intelectual e institucional que le permitirá adaptarse a los códigos de la enseñanza superior para permanecer en el sistema.

Diversas investigaciones (Torcomian, 2015; Carvalho Carneiro \& Rocha Sampaio, 2011; Pérez-Pulido, 2016), nacionales e internacionales, dan cuenta de los cambios que se producen en los estudiantes al ingresar a la educación superior. Torcomian (2015), en una investigación realizada con estudiantes de la carrera de Psicología de la Universidad Nacional de Córdoba, se propone identificar las experiencias en el primer tramo de la carrera universitaria y comprender su vinculación con los procesos de estudio. La investigadora, en este proceso, identifica dos momentos: el primero es durante el cursillo de ingreso, en el que se mantiene una continuidad con la etapa previa, y un segundo momento que los enfrenta a una ruptura en la trayectoria escolar. Refiere al respecto: "Esta ruptura se convierte en una crisis que se deriva en algunos casos en transformaciones de la subjetividad del actor estudiantil, en el modo de operar como así también en la utilización de estrategias durante los procesos de estudio" (p. 38). 
En el trabajo de Carvalho Carneiro y Rocha Sampaio (2011), se abordan las dificultades y los logros de los estudiantes durante su tiempo en la universidad y se establecen relaciones con el proceso de afiliación. Ante los resultados obtenidos, concluyen que la afiliación es solo uno de los pilares que constituye la vida en la universidad y hace que sea posible permanecer en la educación superior. Hay otras dimensiones esenciales para la formación estudiantil, tales como la afiliación intelectual y el establecimiento de interacciones en el entorno académico.

Por su parte, Pérez-Pulido (2016) se propone comprender la manera en que los ingresantes enfrentan los retos y aprovechan las oportunidades que se les presentan durante su proceso de adaptación a la universidad. Se observó que, para responder a las exigencias del campo universitario e incorporar el habitus escolar universitario, el estudiante establece cambios en sus rutinas e invierte capitales económicos y culturales; para ello, se apoya en sus capitales sociales.

En suma, encontramos, en relación a la temática que nos convoca, diversos trabajos que refieren a un proceso de aprendizaje de parte del estudiante para adaptarse a los cambios que se producen en el ingreso a la educación superior. Es decir, los ingresantes se encuentran con el desafío de iniciar la construcción del oficio de estudiante universitario con las particularidades propias del nuevo contexto educativo, proceso que les posibilitará el logro de la afiliación intelectual e institucional para acceder y transitar el primer año en la universidad.

\section{El oficio de estudiante universitario}

El estudiante, para acceder al ámbito universitario y comenzar su trayectoria estudiantil en la institución educativa, tiene que comenzar a construir el oficio de estudiante universitario. Entendemos que este proceso de aprendizaje que lleva a cabo el ingresante en sus inicios es complejo y puede ser abordado desde múltiples perspectivas. En el presente trabajo, para profundizar acerca de esto, tomamos el aporte del sociólogo francés Alain Coulon (1995, 2005), quien acuña el término "oficio de estudiante" para definir este proceso y las dimensiones que lo integran. Al respecto, expresa:

Consideren el nuevo oficio de estudiante como una nueva profesión que ustedes van a ejercer. Esto no solo significa que deberán dedicarle un tiempo importante de su vida inmediata, sino, además es necesario y primordial comenzar con el aprendizaje, dominar los recursos, entender y conocer las reglas. (Coulon, 2005, p. 6)

Dicho de otro modo, aprender el oficio de estudiante universitario consiste en aprender los innumerables códigos que forman parte de la vida académica y demostrar el dominio sobre ellos. Según Coulon (2005), este dominio no solo se manifiesta en los momentos de evaluación académica formal, sino también en interacciones informales en el aula con profesores y otros estudiantes. En este sentido, las prácticas académicas requieren contar con disposiciones y habilidades tales como la expresión oral y escrita; es decir, interpretar el código lingüístico, lo que le va a permitir identificar, interpretar y resolver problemas, presentar -de 
manera precisa- referencias teóricas y bibliográficas, así como la denominada inteligencia práctica.

Lo antedicho da cuenta de que durante el primer año de la universidad, sobre todo en los primeros meses, el ingresante transita un tiempo de verdadero aprendizaje, ya que tiene que reestructurar su habitus y la cultura estudiantil adquirida en la escuela secundaria para desarrollar nuevos esquemas que le posibiliten descifrar e interiorizar la complejidad propia de las prácticas del nivel universitario. En este contexto, cobran relevancia, particularmente, los capitales culturales incorporados como habitus en su trayectoria de vida, desde las prácticas sociales en el seno familiar hasta itinerarios escolares previos. Al respecto, Bourdieu y Passeron (2013) refieren:

Los estudiantes más favorecidos no deben sólo a su medio de origen hábitos, entrenamientos y actitudes que les sirven directamente en sus tareas académicas; heredan también saberes y un saber-hacer, gustos y un "buen gusto" cuya rentabilidad académica, aun siendo indirecta, no por eso resulta menos evidente. (p. 32)

En particular, Coulon (2005) plantea que es posible reconocer si un estudiante ha logrado constituirse en miembro de la institución y formar parte de la cultura institucional cuando puede resolver situaciones prácticas con naturalidad, poniendo en juego patrones de pensamiento o acción, lo que Pierre Bourdieu llamó habitus, que, como un conjunto de pensamientos y prácticas integradas, generan nuevas actitudes y facilita nuevas adquisiciones. Sostiene, al respecto, que las disposiciones mediante las cuales los ingresantes logran incorporar la dinámica propia de la vida universitaria están ancladas en un habitus constantemente renovado que se enriquece con nuevas experiencias que, al internalizarse, se sedimentan en la estructura interna del sujeto.

De hecho, el mencionado autor concuerda con la noción de habitus (Bourdieu, 2007), pero entiende que el concepto de afiliación la completaría y ampliaría, ya que:

La idea de afiliación, que coincide con la idea de habitus desarrollada por Pierre Bourdieu, -pero que a su vez se diferencia de manera clara en que la idea de afiliación está más orientada hacia la actividad del sujeto e indica la confianza que se puede obtener durante la dinámica del aprendizaje en la evolución personal de los individuos-, permite integrar este aprendizaje social activo. (Coulon, 1999, p. 42)

Para complementar la perspectiva de Coulon en relación al proceso de construcción del oficio de estudiante universitario, tomamos el aporte de Bourdieu y Passeron (2013), quienes, desde un enfoque sociodemográfico, refieren al respecto:

La manera más racional de cumplir con el oficio de estudiante consistiría en organizar toda la acción presente con referencia a las exigencias de la vida profesional y en poner en práctica todos los medios racionales para alcanzar, en el menor tiempo posible y lo más perfectamente posible, ese fin explícitamente asumido. (p. 86) 
Es menester aclarar que ambos autores investigan, desde diferentes perspectivas, la ruptura que se produce en los estudiantes, al ingresar a la educación superior, entre lo conocido y el mundo nuevo por conocer. Coulon (1995) particulariza el proceso de aprendizaje que realiza el estudiante para lograr afiliación intelectual e institucional constitutiva del oficio de estudiante; Bourdieu y Passeron (2013) refieren a los códigos que le permitirán el ingreso y la permanencia en el sistema. De igual modo, denominan "oficio de estudiante" al proceso de integración a la vida universitaria y acuerdan en la necesidad del estudiante de incorporar los códigos y reglas institucionales como condición para transitar y permanecer en el sistema.

En síntesis, los conceptos vertidos hasta aquí nos permiten comprender que el oficio de estudiante universitario es un proceso de afiliación intelectual e institucional que le permite al ingresante integrarse a la institución educativa, transitarla y permanecer en ella. Poder ejercer el oficio de estudiante requiere de un proceso de aprendizaje que, según enuncia Coulon (1995), se completa en tres etapas:

El tiempo de alienación; el estudiante entra en un universo desconocido, una nueva institución que rompe con el mundo familiar que acaba de dejar atrás;

el tiempo de aprendizaje; el estudiante se adapta progresivamente a la situación y la asume;

por último, el tiempo de afiliación; período en que se observa ya un relativo dominio, que se manifiesta especialmente por la capacidad de interpretar las reglas, e incluso transgredirlas. (p. 159)

\section{Tiempo de alienación o tiempo de extrañamiento}

Comenzar a transitar la vida de estudiante universitario e integrarse en la institución educativa es un proceso de aprendizaje cuya duración y dificultades dependen de las características institucionales y de la trayectoria familiar y escolar previa del ingresante. Es una experiencia novedosa y de extrañamiento, ya que el conocimiento, el lenguaje y los procedimientos institucionales están organizados de manera diferente a los del nivel secundario. Esta ruptura con lo conocido y el choque con lo nuevo pueden generar, en los estudiantes, nostalgia por los vínculos sociales y la modalidad de relación propia del nivel anterior (Coulon, 2005).

Desde los primeros contactos con las diferentes dependencias institucionales, el estudiante realiza un trabajo de interpretación que le permite comenzar a familiarizarse con este nuevo mundo y construir progresivamente intercambios sociales para solicitar información, inscribirse, adquirir el material de estudio, conocer la pertinencia de las áreas de acuerdo a sus necesidades, etc. Estas situaciones pueden generar sentimientos encontrados; por un lado, el desconcierto y la desorganización por el choque abrupto que se produce al inicio para cumplimentar los trámites e incorporar reglas que desconoce y, por otro, la satisfacción y el entusiasmo por iniciar la carrera universitaria elegida. 
En las instancias iniciales de reconocimiento e integración en la vida académica, se pone en juego la capacidad de autogestión del estudiante para manejarse y resolver aspectos administrativos, así como las dificultades para la organización de los horarios, la elección de materias a cursar y comisiones de cursado, la interpretación del reglamento de enseñanza, etc. Este momento iniciático se constituye en una situación vital para el ingresante; en relación a esto, Coulon concluye:

Estos primeros momentos parecen ser, para la inmensa mayoría de los estudiantes, muy desestabilizadores. Para llegar a ser estudiantes competentes, deben alcanzar un cierto número de nuevos aprendizajes: algunos relacionados con la afiliación institucional; otros, de orden cognitivo, que pueden agruparse dentro de la afiliación intelectual. (p. 110)

\section{Tiempo de aprendizaje}

Después del ingreso a este nuevo contexto educativo, marcado por rupturas y cambios abruptos, el estudiante entra, según Coulon (2005), en un período de latencia en el que dispone de referencias más estables y en el que comienza a aprender los rudimentos del oficio, transformándose en aprendiz. Comienza a familiarizarse progresivamente con los códigos institucionales y a profundizar el trabajo intelectual.

En esta etapa, los estudiantes ponen en juego su modalidad de aprendizaje, los recursos y disposiciones incorporados (habitus) en la trayectoria familiar y escolar para elaborar estrategias que le permitan llevar a cabo el trabajo académico. Coulon (2005) expresa acerca de las estrategias que los estudiantes construyen a partir de una autoevaluación personal basadas en el habitus:

No se trata de estrategias conscientes. Son tan aceptadas como calculadas: la práctica de la estrategia incorpora estructuras familiares, situaciones socialmente estructuradas y, al mismo tiempo, depende de operaciones cognitivas que toman en cuenta la situación actual y su "sentido práctico" de las normas. (p. 123)

Lo referido por Coulon es consistente con la conceptualización de estrategia planteada por Bourdieu, quien la define, de manera imbricada con el habitus, como disposiciones para la acción de acuerdo al lugar que cada agente ocupa en el entramado social y de acuerdo a las situaciones que se le presentan, lo cual le otorga la posibilidad de cambio. De esta manera, expresa: "Hablo de estrategias que son acciones objetivamente orientadas con fines que pueden no ser los fines subjetivamente perseguidos" (Bourdieu, 1984, p. 119).

Los conceptos de Coulon y Bourdieu nos habilitan a sostener que las estrategias desplegadas por los estudiantes para el aprendizaje del trabajo universitario posibilitarán la afiliación intelectual del ingresante. Una vez lograda, podrá comprender las instrucciones, a menudo implícitas, de los profesores para llevar a cabo los trabajos académicos. El descubrimiento de estas recomendaciones docentes, naturalizadas en las prácticas de educación superior, es una de las prime- 
ras tareas que el ingresante debe llevar a cabo para convertirse en un aprendiz de estudiante. Asimismo, necesita vislumbrar las diferentes categorías que componen el plan de estudio (sistema de regularidad y aprobación, correlatividades, etc.), comprender las exigencias del trabajo intelectual, trabajar de manera autónoma y tomar conciencia del tiempo personal para la consolidación de los aprendizajes.

Estos aprendizajes, que se consideran adquiridos por la cultura universitaria, derivan, en este momento del proceso de integración a la vida académica, en que algunos estudiantes se planteen el cambio de carrera por entender que no es lo que esperaban o en que abandonen los estudios por no poder integrarse académicamente a la institución. También surge, en quienes tienen dificultades para establecer vínculos sociales, el sentimiento de anonimato, de desolación, porque no logran integrarse o sostener un grupo de amigos estable, lo cual dificulta la permanencia y continuidad de los estudios.

\section{Tiempo de afiliación}

En el tiempo de afiliación, el estudiante comienza a familiarizarse progresivamente con el nuevo ambiente educativo. Coulon expresa acerca de esta etapa:

El estudiante está entonces doblemente afiliado: por un lado, en el plano institucional, él sabe a partir de ahora comprender e interpretar los múltiples dispositivos institucionales que rigen la vida cotidiana de un estudiante; por otro lado, comienza a conocer de la misma manera lo que se espera de él, en el plano intelectual esta vez, para que demuestre su competencia de estudiante. Esta segunda forma de afiliación, que se inicia hacia el final del primer semestre, nunca se acaba, se debe recomenzar y confirmar permanentemente, mientras que la primera se adquiere de manera definitiva. (p. 155)

En otras palabras, podemos decir que el estudiante logra dos tipos de afiliación: la afiliación institucional, que refiere al conocimiento de los modos de vinculación y funcionamiento de la universidad, es decir, la cultura institucional, así como también los códigos y normas que la regulan; y la afiliación intelectual, que implica que el estudiante, desde el terreno de lo conceptual, pueda interpretar reglas y consignas, comparta el lenguaje común del grupo y las prácticas propias de la esfera de la educación universitaria y les otorgue sentido.

De esta manera, el sentido práctico y la temporalidad de la regla son dos propiedades constantemente presentes en la acción práctica (Coulon, 2005). Las reglas institucionales que marcan la vida estudiantil forman parte de la cultura institucional, existen, están presentes, pero su eficacia, su existencia concreta, es una práctica de activación. Esto se debe a que la interpretación de estas reglas es realizada por los estudiantes en el curso de sus interacciones. Los estudiantes tienen que pesquisar la organización social de las reglas y analizar cómo y de qué manera se pueden transformar en acción práctica. De igual modo, decir que una regla es considerada por los actores como una cuestión práctica no significa que 
sea clara y fácilmente comprensible, ya que -a veces- se presentan ambigüedades y desacuerdos entre los que se encargan de aplicarla.

A su vez, en el discurso académico y en el trabajo intelectual, existen códigos disimulados que tienen que ser identificados e interpretados por los estudiantes para lograr la afiliación, por tanto, la construcción del oficio de estudiante. Coulon (2005) presenta algunos marcadores que dan cuenta de que esto ha sido logrado:

- En su forma institucional, la afiliación se lleva a cabo cuando el estudiante ha adquirido suficiente familiaridad con las reglas para no cometer errores, puede reconocer sus excepciones y es capaz de identificar y usar naturalmente estos códigos en su práctica cotidiana. En resumen, cuando entiende las reglas del plan de estudios y las cuestiones prácticas que necesita para resolver las situaciones de la vida estudiantil.

- Con respecto a las formas propiamente intelectuales de afiliación, estas consisten en la comprensión del tipo de trabajo intelectual solicitado y la posibilidad de llevarlo a cabo. Esto es otorgarle el tiempo y los recursos necesarios para resolver lo solicitado de manera correcta a nivel formal y conceptual.

En este momento del proceso, el estudiante adquiere la capacidad de transformar las reglas en acciones prácticas. Cuando logra aprenderlas y resignificarlas, pasa a ser miembro afiliado de la comunidad educativa. "Afiliarse, por lo tanto, es naturalizar por la vía de la incorporación las prácticas y la dinámica universitaria, que no existen previamente en los hábitos estudiantiles" (p. 160).

Es decir, la afiliación no se reduce a la exposición de formas ritualizadas de conocimientos, implica haber internalizado el campo de códigos propios del ámbito académico para poder dedicarse a los conocimientos y conceptos, objeto principal del trabajo intelectual. Superada esta primera etapa de afiliación intelectual, donde los marcadores intelectuales han sido objeto de una verdadera incorporación, el estudiante tendrá la tarea de diseñar innovaciones, variaciones y creaciones que le darán acceso a la investigación científica.

\section{Metodología}

En función de nuestro problema de investigación, se optó por el modelo metodológico cualitativo. Entendemos que este enfoque supone una forma de considerar y mirar el objeto de estudio en la que cobran relevancia la perspectiva de los actores protagonistas de la acción social y el contexto en el cual se lleva a cabo la investigación. Asimismo, nos posibilita acceder a los sentidos y significados que los sujetos, en este caso los ingresantes, le atribuyen a sus acciones, respetando la singularidad de sus trayectorias. Particularmente, procuramos reconocer, desde las voces de los protagonistas, con qué capitales culturales y escolares cuenta el ingresante migrante a la Facultad de Ciencias Químicas e interpretar cómo estos capitales se relacionan con la construcción del oficio de estudiante. 
Para la recolección de datos, se eligió la entrevista cualitativa en profundidad, ya que consideramos que esta estrategia nos permite establecer un vínculo con los entrevistados que se va afianzando en los sucesivos encuentros, donde la escucha y la observación posibilitan comprender la mirada que los actores sociales tienen de la situación objeto de investigación (Taylor \& Bodgan, 1987).

Se trabajó, en el transcurso de un año lectivo, con una muestra constituida por catorce estudiantes migrantes, ingresantes a la Facultad de Ciencias Químicas de la Universidad Católica de Córdoba y de la Universidad Nacional de Córdoba. Con la intención de caracterizar el grupo de estudiantes, se presenta a continuación un cuadro en el que se especifica edad, sexo, ciudad desde donde han migrado para radicarse en Córdoba capital e institución en la que iniciaron sus estudios (ver Cuadro 1).

Como se observa en el Cuadro 1, de los catorce entrevistados, cuatro son varones y diez, mujeres. Tres varones y una mujer están cursando en la Universidad Católica; el resto, en la Universidad Nacional. Se encuentran en la franja etaria de diecisiete a veintidós años, esto da cuenta de que la mayoría son estudiantes que recién finalizan el nivel secundario y, en este momento, están transitando sus primeras experiencias en el ámbito universitario.

Cuadro 1: Caracterización de los estudiantes de la muestra

\begin{tabular}{|c|c|c|c|l|}
\hline Estudiante & Edad & Sexo & Universidad & Ciudad de origen \\
\hline$A$ & 19 & $F$ & UNC & Río Turbio - Santa Cruz \\
$B$ & 17 & $F$ & UNC & Santiago del Estero \\
C & 18 & $M$ & UNC & Rafaela - Santa Fe \\
$D$ & 20 & $F$ & UNC & Santiago de Chile (Chile) \\
$E$ & 18 & $F$ & UNC & Las Marcas (Italia) \\
$F$ & 18 & $F$ & UNC & Balnearia - Córdoba \\
$G$ & 17 & $F$ & UNC & Federal - Entre Ríos \\
$H$ & 20 & $F$ & UNC & Catamarca \\
$I$ & 18 & $F$ & UNC & Santiago del Estero \\
$J$ & 18 & $F$ & UNC & La Carlota - Córdoba \\
$K$ & 17 & $M$ & UCC & Catamarca \\
L & 22 & $M$ & UCC & Colombia \\
$M$ & 17 & $M$ & UCC & Concepción del Uruguay - Entre Ríos \\
$N$ & 18 & $F$ & UCC & Rufino - Santa Fe \\
\hline
\end{tabular}




\section{Resultados ${ }^{2}$}

A continuación, presentaremos algunos hallazgos de la investigación que nos permiten visualizar cómo los estudiantes transitaron el proceso de afiliación institucional e intelectual en la construcción del oficio de estudiante.

Al indagar sobre las primeras percepciones y experiencias en el nuevo contexto educativo, algunos estudiantes que se encuentran cursando en la Universidad Nacional valoran positivamente el clima institucional, la cercanía con los profesores $u$ otros actores institucionales al comparar, con vivencias propias o con discursos de otros jóvenes, la Facultad de Ciencias Químicas con otras unidades académicas de la Universidad.

"No hablo de la universidad, más de la facultad y me parece como que esta facu creo que al ser un poquito más chica que otras es como que tiene un ambiente distinto". (C.1)

"Me ha parecido muy bueno y muy diferente el trato de la facultad hacia los alumnos que en otras facultades. Muy diferente y muy bueno". (I.1)

Suponemos que estas primeras percepciones son posibles porque al ingresar a la facultad, los primeros dos meses, cursan la asignatura Introducción al estudio de las Ciencias Químicas (ciclo de nivelación) en comisiones que tienen entre diez a treinta estudiantes y con un docente guía, único referente en esa etapa. El espacio áulico, donde el intercambio con el docente es directo y permanente, así como la presentación de diferentes programas de la facultad (becas y tutorías) les posibilita percibir que la institución procura múltiples oportunidades de inclusión.

De igual modo, durante el transcurso del año, los estudiantes continúan destacando las acciones implementadas en la facultad que facilitan el cursado, como -por ejemplo- el hecho de no tener clases las dos semanas de parciales.

"A mí me sorprendió mucho que nos den dos semanas libres". (H.2)

"A mí me encantaron las dos semanas esas que nos dieron sin clases para ren$\operatorname{dir}^{\prime \prime} \cdot(\mathrm{J} .2)$

Por otro lado, en el relato de algunos estudiantes, se percibe lo dificultoso que les resulta la integración a la vida académica en relación a las nuevas demandas y a cuestiones prácticas de cursado como inscripciones y carga horaria.

"Es muy linda pero me cuesta bastante". (B.1)

"El único problema son los horarios que bueno por la comisión, por la parte del guaraní que es tan difícil que tenés que inscribirte, que cierra, abren los horarios y todo lo demás". (E.2)

"Lo que me cansa mucho no es el estudio sino la carga horaria que estamos teniendo". (J.2)

\footnotetext{
2 En este apartado, a los fines de aclarar los resultados, se incorporan pequeños extractos de las entrevistas realizadas a los estudiantes. Las siglas corresponden al modo en que fueron nombrados para mantener el anonimato y el número corresponde al orden de la entrevista.
} 
Estas expresiones dan cuenta de prácticas institucionales que forman parte de la vida estudiantil: interpretar las reglas y códigos propios del ámbito universitario y llevar a cabo acciones que les permitan hacer uso de los dispositivos institucionales para conseguir lo que se proponen, más allá de las dificultades propias del sistema. Una estudiante expresa: "Me había tocado una a la noche y estuve toda la mañana actualizando la página, actualizando la página y se ve que alguien se bajó de la que yo quería... de suerte, no sé tuve suerte y rápido". (J. 3)

De igual modo, en relación a la vida estudiantil, surge, en las entrevistas realizadas al finalizar el año, el cansancio que sienten los ingresantes por haber iniciado el cursado a finales de enero, fecha en que comenzó el ciclo de nivelación en la Universidad Nacional, y por la energía puesta en adaptarse a lo novedoso del ámbito universitario.

"Creo que... o sea, fue cansador acostumbrarse a un montón de cosas durante un año y estudiar tanto durante un año". (C.4)

"Con ganas de que termine ya ino aguanto más!... se me hizo muy largo". (J.4)

"iQué cansancio!... iYa quiero terminar ya!". (G.4)

Al consultarles a los ingresantes de la Universidad Católica sobre sus primeras experiencias en la institución, manifiestan estar cómodos y a gusto en la facultad: "La facu en general me gusta" (N.1); "Me resulta bastante práctico el ámbito de la facultad, o sea no tengo ningún problema, los profesores son bastante buenos" (K.1).

También refieren a cuestiones organizativas de la institución. Un estudiante menciona: "es medio complicado el tema que nos cambian las fechas de los parciales de un día para el otro que cambia, que vuelve, que eso bueno, todavía me estoy acostumbrando" (K.1). Otro, en cambio, expresa: "Salvo muy pequeñas excepciones tenés los horarios re bien estructurados así que tenés que venir te quedas las seis horas que te tenés que quedar y te vas, no tenés que volver más" (M.2). Ambos refieren a aspectos diferentes de las prácticas institucionales, pero estrechamente vinculados con la cultura propia de la facultad.

Los relatos estudiantiles nos permiten visualizar que las prácticas académicas propias del nivel universitario presentan, para el ingresante, experiencias novedosas que tienen que ser decodificadas por el estudiante e integradas a sus conocimientos previos para poder otorgarle significación y sentido. Asimismo, las nuevas experiencias posibilitan complejizar los esquemas de acción previos y construir otros esquemas para dar respuesta a la complejidad de las prácticas inherentes a las trayectorias académicas universitarias.

En este sentido, si consideramos el relato de los ingresantes en relación a la trayectoria educativa previa, los estudiantes que se describen como regulares, con dificultades particularmente en el área de las matemáticas, cuando se les pregunta sobre cómo les ha ido en el primer trayecto en la universidad, evidencian un recorrido esforzado:

"Me cuesta bastante pero bueno, lo estoy intentando. Y no sé si me va a ir bien, pero lo estoy intentando". (B.1) 
"Me saqué un tres, tengo que recuperar ahora y sacar un seis ahora en el segundo". (D.1)

Algunos de estos ingresantes no lograron regularizar ni aprobar el ciclo de nivelación; otros pudieron regularizarlo para iniciar el primer cuatrimestre, pero no sostuvieron el cursado e interrumpieron su trayecto a mitad de año. Esto nos permite suponer que los capitales internalizados en su trayectoria de vida no se constituyeron en un aporte sustantivo para la construcción de conocimientos y saberes previos acerca de las prácticas habituales del campo universitario, lo que les permitiría posicionarse con mayor seguridad ante lo novedoso que les propone este tiempo de aprendizaje (Coulon, 2005). Esto genera disonancias entre lo que la institución supone adquirido y el habitus de los estudiantes.

Por otro lado, entre los que refieren haber tenido calificaciones altas en su escolaridad previa, una parte de ellos dice que les va bien.

"Me saqué en Matemática un ocho, en Química un nueve, en Física un siete y en Laboratorio un ocho". (C.2)

"En Química y en Laboratorio bien, muy bien. Y en Física y Matemática, ahí, no las he podido aprobar, digamos, no he pasado del seis, pero he estado cerquita". (I.2)

"Me saqué un nueve en todas menos en Laboratorio que me saqué un ocho y ahora estoy para los segundos". (J.2)

Algunos de estos estudiantes parecieran haber internalizado con facilidad, en el trascurso del primer cuatrimestre, la dinámica particular de las prácticas académicas e institucionales. Han obtenido un buen desempeño académico y reconocen e interpretan los códigos propios del campo universitario. Esta doble afiliación (académica e institucional) nos permite suponer que se encuentran, a mitad de año, transitando el tiempo de afiliación propuesto por Coulon (2005), como última etapa en la construcción del oficio de estudiante.

Estos logros posibilitaron la toma de decisiones en cuestiones vinculadas con el cursado del segundo cuatrimestre. Los estudiantes de la Universidad Nacional decidieron sumar, a las asignaturas establecidas por el plan de estudio, otras asignaturas complementarias, Inglés e Informática, materias que se pueden cursar en cualquier momento de la carrera. Un estudiante de la Universidad Católica está analizando la posibilidad de sumarse a un equipo de investigación como colaborador.

"Me anoté en Inglés no más... total las puedo hacer cuando pueda en vez de cargarme tanto me anoto en Inglés y el otro cuatrimestre me anoto en Informática". (J.3)

"Me inscribí para hacer Inglés y me inscribí en Informática pero se me superponen los horarios". (G.3)

"Este semestre estaba la posibilidad de comenzar digamos aunque sea digamos en un proyecto de acá de la facu pequeño". (K.2)

Podríamos considerar, entonces, que este grupo de estudiantes ha puesto en juego las estrategias necesarias que les permiten llevar a cabo el trabajo intelec- 
tual y la organización de sus tiempos para dar respuesta a las demandas académicas. De modo tal que algunos se plantean formar parte de grupos de tareas más amplios como los equipos de investigación y otros, habiendo logrado el reconocimiento de los códigos del nivel superior, en este caso, las reglas propias del plan de estudio, han decidido adelantar asignaturas.

Otro grupo de ingresantes, en relación al desempeño esperado por ellos, ha sido medianamente sorprendido por los resultados alcanzados. En estos casos, depende de las expectativas en relación al cursado cómo vivencian el no haber logrado el resultado esperado:

"Pensé que me iba a ir mejor, pero bueno, me tendré que preparar más para el próximo". (F.1)

"No promocioné ningún parcial pero me fue relativamente bien así que bueno, por lo menos me sirve como motivación de no haberme sacado un uno". (H.2)

"Pero que a veces como que no sirve porque de las cuatro materias promocioné dos y las otras dos no y como que... fue una ducha de agua fría digamos porque yo re confiaba de que me había ido bien". (E.2)

El grupo de estudiantes antes mencionado ha evidenciado, a lo largo del año, la necesidad de implementar mayores esfuerzos subjetivos y cognoscentes para sostener el cursado por cuestiones aparentemente vinculadas a la interpretación de los códigos y las reglas propias de las prácticas académicas e institucionales.

Una de ellas solo cursó, en el segundo cuatrimestre, las asignaturas complementarias (Inglés e Informática) porque en una de las asignaturas centrales del primer cuatrimestre quedó libre y esto le impidió inscribirse en las del segundo.

" $Y$ eso me trabó el segundo cuatrimestre porque intenté rendir la general pero como la rendí libre me dijeron que califican de una forma diferente libre. Eso no sabía yo cuando fui a rendir"; "el tema de la regularidad también es, no sé si complicado pero con los parcialitos y eso a mí me iba bien en los parciales pero bueno... por el tema de los parcialitos y eso quedé libre" (H.3).

En sus relatos se refleja claramente que todavía no logró entender cómo es el funcionamiento del reglamento de enseñanza, particularmente lo referido a los requisitos necesarios para acceder a las condiciones de cursado y aprobación.

Otra estudiante pudo regularizar las asignaturas del primer cuatrimestre y está cursando todas las del segundo. En una de las entrevistas realizadas antes de finalizar el primer cuatrimestre, manifestaba lo siguiente:

"Me conviene sentarme directamente y probar hacer todo, intentar hacer todo y tener la posibilidad de recuperatorio porque ir a final significa estudiar todo y confiar en ese único parcial que si me anda mal tengo que esperar hasta diciembre..., entonces como que utilizo todos los recursos que me ofrecen y si ando bien, bien y si ando mal tengo la opción de recuperar" (E.2).

Aquí hace referencia a su intención de rendir los segundos parciales y recuperar los primeros en un plazo de dos semanas. Si no lograba aprobar, rendiría los finales en la semana siguiente. Esto da cuenta de que no alcanza a comprender la 
lógica académica y el manejo de los tiempos como para poder realizar una planificación sobre su cursado que le permita lograr los resultados que se propone. De hecho, no logró aprobar en ninguna de las instancias. Esta dificultad para la toma de decisiones se reitera al finalizar el año:

"En la primera mesa en diciembre hago toda lo uno y si las apruebo es como que la segunda mesa en diciembre me da cosa dejar la opción que yo pueda rendir y pasarla directamente en febrero y puede ser que en febrero me ande mal" (E.4).

Considero que, en esta modalidad particular de resolver las situaciones académicas, intervienen dos cuestiones: por un lado, la creencia de que aprovechar todas las instancias de examen le garantiza mayores posibilidades; por otro, cuestiones vinculadas a su estructura subjetiva, ya que el deseo de llevar la carrera al día y la ansiedad que esto le genera interfieren en sus esquemas de acción para realizar un análisis de la situación que contemple todas las variables en juego y le permita tomar una decisión acorde a las posibilidades reales de concreción.

Esta estudiante, oriunda de Italia, que en reiteradas oportunidades expresó frustración por su desempeño académico, encuentra en el idioma una barrera para comprender el tipo de trabajo intelectual solicitado y resolverlo de manera correcta a nivel formal y conceptual:

"Porque me equivoqué en un Verdadero y Falso y como que, yo pensaba que había puesto bien pero por una palabra, que hace cambiar el significado de todo... como que no se me terminaba de aclararme lo que me pedía el enunciado". (E.2)

"Porque yo me había equivocado en ejercicios porque no había entendido qué me estaba pidiendo y no, no se puede preguntar al profesor... hice lo que yo había entendido y no era nada que ver". (E.3)

En síntesis, ambas estudiantes, que se encuentran en proceso de construcción del oficio de estudiante universitario, atravesando el tiempo de aprendizaje (Coulon, 2005), gestionan de manera autónoma su propia trayectoria, pero al no comprender de modo cabal los códigos y reglas inherentes a las prácticas, se encuentran limitadas para pensar estrategias que les permitan abordar la situación y construir soluciones acertadas. Aún más la estudiante italiana, que tiene insuficiente dominio del idioma, en cuyo caso la alfabetización académica se complica, ya que las prácticas académicas que requieren interpretar el código lingüístico para identificar, comprender y resolver problemas se encuentran limitadas.

Lo hasta aquí referido habilita a pensar que, cuando se trata de trayectorias estudiantiles, los alumnos valoran el desempeño académico en términos de los resultados obtenidos en los exámenes. Pareciera naturalizado socialmente que el ingreso a la universidad requiere el pasaje, al decir de Frigerio (2017), de un "umbral" entendido como frontera con guardia, portero, en el que las distintas modalidades de evaluación para el acceso y durante el cursado ofician de garante para custodiar aquellos saberes reservados solo para algunos.

Los estudiantes reconocen este escenario y manifiestan, en mayor o menor medida, estrés y ansiedad ante la situación de examen. Relatan que estos sentimientos producen tensiones en las estructuras afectivo-emocionales al punto de 
generar interferencias en los aspectos intelectuales que limitan la posibilidad de dar cuenta de todo lo que saben. Asimismo, se advierte cómo regulan sus emociones de acuerdo a ciertas representaciones o ideas previas acerca de lo que "se debe" o "se puede" hacer por estar en la universidad.

"Fue re frustrante (risas) fue re feo... Me sentí re mal, encima estaba en el aula cuando vi la nota, yo ya presentía que me iba a ir mal, cuando vi la nota como que me dieron ganas de llorar y dije no, estás en la universidad no podés llorar". (G.1)

"Tal vez en el examen me puse un poco nervioso... en una parte me puse bastante nervioso". (C.2)

"El miedo que tenía yo por de la ansiedad, de la agitación nerviosa porque era, realmente tenía nervios". (E.1)

Por otro lado, en relación a los exámenes, uno de los estudiantes expresa: "Algunos exámenes que sabés como que a veces los hacen como con alguna trampita para que vos pises el palito, pero bueno, no me parecieron nada del otro mundo los exámenes tampoco" (C.2). Se visibiliza en esta expresión el reconocimiento de aquellos códigos que suelen ser disimulados en las prácticas académicas, en este caso los exámenes, que tienen que ser identificados e interpretados por los estudiantes para lograr los resultados esperados.

En la misma línea de análisis, se identificaron, en los discursos, algunas diferencias entre las formas de organizar el estudio en la escuela secundaria y los requerimientos necesarios para responder a las nuevas demandas de la universidad. Uno de los puntos más significativos en los que se evidencia esta diferencia está en la anticipación del tiempo necesario que deben invertir para llegar de mejor manera a las instancias evaluativas. La comparación que ellos hacen se ejemplifica con el hecho de que, en la escuela secundaria, con una lectura previa el día anterior era suficiente:

"Antes veía la carpeta y ya está, me iba bien. No es como aquí que tienen que estar horas y horas estudiando". (B.1)

"Yo con leer ya estaba y era un día antes y eso es un mal hábito porque en la facultad con un día antes no llego". (H.1)

"Yo en el secundario estudiaba la noche anterior y me sacaba un diez". (J.2)

Respecto al modo de organizarse para estudiar, pareciera que algunos estudiantes requieren de un período de tiempo para incorporar a su habitus nuevas actitudes y disposiciones que les permitan lograr el ritmo de estudio que se requiere para responder a la dinámica propia de la vida académica universitaria:

"Pero igual por ejemplo al no estar acostumbrada a llevar un ritmo de estudio así de todos los días, eso me costó implementar, me re costó sentarme a estudiar todos los días... ahora sé qué tiempo dedicarle a lo práctico y a lo teórico sería, hasta eso sé". (G.2)

"Todos los días me pongo a hacer ejercicios, pero el hecho de estudiar, no sé... no sé... me costó, me costó, me costó, pero por suerte lo agarré rápido". (J.2)

"Me cuesta mucho y estudio, estudio, tengo que estudiar como cuatro veces más que las otras personas porque si no, no rindo bien". (D.1) 
Otro de los aspectos en los que se establece comparación con el secundario es en la forma de estudio y en las estrategias empleadas para estudiar. En la mayoría de los casos, los estudiantes, para hacer frente a los requerimientos académicos, tuvieron que desplegar las disposiciones y recursos con los que contaban y enriquecer estas disposiciones previas con nuevas estrategias:

"Antes en el secundario y eso estudiaba no sé, de libros y eso y no puedo estudiar yo de los libros en sí y ahora como que empecé a hacer resúmenes y resaltar lo más importante $y$ de ahí a estudiar". (A.2)

"Antes, hacía solamente el fin de semana que acumulaba y me dividía las cosas... mientras que como ahora utilizo cualquier tiempo durante la tarde a poder hacer algo en sí a lo largo del día. Ejercicios, más ejercicios y además no sólo el ejercicio del cual rendí sino también que están por ejemplo o en los libros o en Internet". (E.2)

"Y a mí lo teórico no tengo dificultad de aprenderlo entonces le dedico el ochenta por ciento del tiempo a lo práctico que es lo que más me cuesta y después sí, me pongo con lo teórico". (G.2)

Estos relatos visibilizan cómo los estudiantes han tenido que realizar ciertas modificaciones en su modalidad de aprendizaje para lograr aprender en tiempo y forma los contenidos curriculares. Las estrategias utilizadas refieren a dos cuestiones puntuales. Por un lado, el manejo y la administración del tiempo de estudio; el currículo de primer año, en ambas facultades, tiene mucha carga de contenido práctico que -a su vez- tiene correlato teórico. Esto hace que los estudiantes tengan que dividir su tiempo para la resolución de ejercicios y para estudiar la teoría y dedicar mayor carga horaria a la actividad que más les cuesta. Por otro, la multiplicidad de recursos para lograr esos aprendizajes, entre otros, realizar resúmenes, leer y estudiar de los libros.

En otro sentido, pero directamente vinculado con lo que se viene desarrollando, surgen, en relación al estudio, cuestiones que refieren a la lógica disciplinar específica, es decir, a la alfabetización académica. Algunos estudiantes reflejan en sus relatos dificultades para adaptarse al lenguaje disciplinar de las ciencias químicas. Al respecto, Carlino (2002) expresa: "la lectura y escritura exigidas en el nivel universitario se aprenden en ocasión de enfrentar las prácticas de producción discursiva y consulta de textos propias de cada materia" (p. 6).

"Yo antes estudiaba, qué se yo... tenía materias como Economía o Derecho y tenía que estudiar como decía el texto, básicamente, de alguna forma memorizar mucho... acá es mucho, digamos, mezcla de práctica y teórica entonces es como que no sé si vale tanto la pena estudiarte tanta teoría". (C.2)

"Digamos me aprendo las imágenes y todo eso me lo memorizo lugares y eso. Pero cuando me ponen a aprender nombres y muchos nombres y esas cosas me resulta un poco difícil". (L.1)

"Vos tenés que entender el concepto, no tanto el método para desarrollarlo, así que, la única que me permiten ofrecerme el concepto son los libros o Internet. (E.2) 
Finalmente, hacemos referencia a algunos relatos estudiantiles en los que refieren a "otros" que contienen, acompañan y favorecen la integración a la vida universitaria y el proceso de aprendizaje. Otros del núcleo cercano (padres, amigos, novios), externos a la facultad (academia, profesores particulares) o actores institucionales (compañeros, tutores):

"Mi novio que él también... él estudia ingeniería, y él también me ayuda mucho, así que... en el sentido de ponerme a estudiar". (I.2)

"Me junté bastante con una amiga y bueno con ella estudiamos, fuimos con una profe y bueno lo fuimos manejando". (H.2)

"Fui a una clase a un profe particular para aclarar algunas cosas que tenía muchas dudas". (C.2)

"Yo creo que ellos (padres) me re incentivan porque por ahí estoy media bajón, pero ellos desde allá siempre me están ayudando". (G.2)

\section{Conclusiones}

Lo hasta aquí expresado nos habilita a pensar que el comienzo de la vida universitaria les ha supuesto a los ingresantes cambios en relación al rol de estudiante ejercido en instancias de escolaridad previa, es decir, el aprendizaje del oficio de estudiante universitario con las connotaciones y particularidades propias del ámbito académico y de la institución en la que inician sus estudios.

En este sentido, si atendemos al interrogante sobre las prácticas y acciones que despliega el estudiante para ejercer el oficio de estudiante universitario, los relatos dan cuenta de que, para responder a las demandas universitarias, han tenido que poner en juego todos los recursos con los que contaban y enriquecer estas disposiciones previas con nuevas estrategias. Para la mayoría de los estudiantes, el despliegue del capital cultural incorporado fue suficiente para obtener un buen desempeño en toda su trayectoria por el primer año y lograr los resultados pretendidos. Sin embargo, otros que aún no lograron la alfabetización académica -es decir, interpretar la lógica propia de las prácticas inherentes al campo disciplinar específico- sienten frustración y decepción porque los esfuerzos resultaron insuficientes y los resultados distan mucho de lo que hubieran pretendido.

Es decir, contar con los capitales culturales y escolares internalizados en la trayectoria de vida pareciera no ser suficiente para el logro de resultados académicos. Los estudiantes tienen que poder interpretar las reglas y códigos de este campo e integrar a su habitus nuevos aprendizajes y modos de comprender la realidad que les permita gestionar de manera autónoma su trayectoria estudiantil. Al respecto, Coulon (1995) refiere que esta tarea implica un aprendizaje práctico que permita la incorporación, a las estructuras del sujeto, de "nuevos métodos de comprensión" para interpretar el nuevo contexto. En el transcurso del año, a medida que las exigencias académicas fueron aumentando y los contenidos adquirieron cierto grado de complejidad, los estudiantes debieron reestructurar su modalidad de aprendizaje e incorporar nuevas disposiciones para hacer frente a las demandas académicas. 
En esta línea, podemos dar respuesta a la inquietud acerca de los cambios que realiza el estudiante en la forma y los recursos que utiliza para estudiar. Al respecto, todos los entrevistados refieren haber tenido que realizar modificaciones en su modalidad de aprendizaje. Los primeros cambios hacen referencia al tiempo que dedican al estudio. En comparación con trayectos previos, ahora deben disponer de mayor carga horaria y sostener un ritmo de estudio durante el cursado, que se intensifica en épocas de exámenes. Asimismo, refieren a la forma de organizar el estudio. El transcurrir del año les posibilitó a la mayoría comprender la lógica de las prácticas académicas y el alcance de sus posibilidades y limitaciones; a partir de esto, pudieron elaborar -de manera estratégica- un plan de estudio para priorizar los contenidos teóricos o prácticos de acuerdo a sus necesidades.

Por otro lado, dan cuenta de que, para responder a las demandas universitarias, han tenido que poner en juego todos los recursos con los que contaban y enriquecer estas disposiciones previas con nuevas estrategias, entre las cuales mencionan: descargar libros, realizar mapas conceptuales y resúmenes, tomar apuntes, practicar ejercicios, estudiar en grupo. Esto nos permite inferir que una modalidad de aprendizaje flexible y permeable a lo novedoso facilita la integración de las nuevas experiencias y les otorga sentido en función de los conocimientos previos. De este modo, las nuevas incorporaciones enriquecen y posibilitan la reestructuración de las disposiciones y esquemas del sujeto para dar respuesta a las situaciones problemáticas, pensar estrategias y construir posibles soluciones.

Sobre el cierre, compartimos algunos interrogantes que surgen a partir del análisis realizado en el proceso investigativo y que nos permiten seguir interpelando nuestro quehacer profesional cotidiano: ¿qué disposiciones subjetivas, cognitivas y sociales son favorecedoras de estos aprendizajes?; ¿en qué medida los "otros" (padres, compañeros, amigos, profesores) que sostienen acompañan y brindan la contención emocional y simbólica para que esto suceda? y ¿qué lugar ocupa la institución universitaria en el proceso de construcción del oficio de estudiante?

Al respecto, quienes transitamos diferentes espacios universitarios observamos, con preocupación, que las acciones institucionales que se implementan para acompañar a los estudiantes en los primeros años de la universidad no resultan suficientes para fortalecer las trayectorias educativas y favorecer la permanencia. En este sentido, a partir del presente trabajo se propuso, a ambas instituciones, la implementación de uno o varios talleres a los fines de acompañar las trayectorias estudiantiles, particularmente a los estudiantes migrantes en su proceso de integración al nuevo contexto educativo y social. Consideramos que estos espacios de reflexión, escucha y diálogo propician la resignificación de las vivencias propias y en relación a la institución educativa, así como la construcción colectiva de recursos y estrategias que promuevan aprendizajes significativos y favorezcan mejores condiciones para transitar la vida académica. 


\section{Referencias bibliográficas}

Bourdieu, P. (1984). Questions de Sociologie. Paris: Les Editions de Minuit. Recuperado el 12 de agosto de 2018, de: https://monoskop.org/images/4/47/ Bourdieu_Pierre_Questions_de_sociologie_2002.pdf.

Bourdieu, P. (2007). El sentido práctico. Buenos Aires: Siglo XXI.

Bourdieu, P. \& Passeron, J. (2013). Los herederos. Los estudiantes y la cultura. Buenos Aires: Siglo XXI.

Carlino, P. (2002). ¿Quién debe ocuparse de enseñar a leer y a escribir en la universidad? Tutorías, simulacros de examen y síntesis de clases en las humanidades. Lectura y vida, 1(23), 6-14. Recuperado el 20 de junio de 2018, de: https:/ /www.aacademica.org/paula.carlino/91.pdf.

Carvalho Carneiro, A. \& Rocha Sampaio, S. M. (2011). Estudantes de origem popular e afiliação institucional. En S. M. Rocha Sampaio (org.), Observatório da vida estudantil. Primeiros estudos (pp 53-69). Salvador: Editora da Universidade Federal da Bahia. Recuperado el 25 de junio de 2016, de: http://static.scielo.org/ scielobooks/n656x/pdf/sampaio9788523212117.pdf.

Coulon, A. (1995). Etnometodología y educación. Buenos Aires: Paidós.

Coulon, A. (1999). Un instrument d'affiliation intellectuelle. L'enseignement de la méthodologie documentaire dans les premiers cycles universitaires. BBF. Paris. $T$, 1(44), 36-42. Recuperado el 12 de agosto de 2018, de: http://bbf.enssib.fr/ consulter/bbf-1999-01-0036-005.

Coulon, A. (2005). Le Métier d'Étudiant. L'entrée dans la vie universitaire. Paris: Economica.

Frigerio, G. (2017, agosto). Saberes sobre los umbrales: sentidos que se ponen en juego en el ingreso a la universidad. Conferencia del VII Encuentro Nacional y IV Encuentro Latinoamericano "Los sentidos del Ingreso y su lugar en la agenda de las universidades". UNCUYO.

Pérez-Pulido, I. (2016). El proceso de adaptación de los estudiantes a la universidad en el Centro Universitario de Los Altos de la Universidad de Guadalajara. Tesis de Doctorado Interinstitucional en Educación. Guadalajara, Jalisco: ITESO. Recuperado el 10 de agosto de 2018, de: https://rei.iteso.mx/bitstream/handle/11117/ 3591/DIETesis\%20Ignacio\%20P\%C3\%A9rez_copy.pdf?sequence $=5$.

Taylor, S. J. \& Bodgan, R. (1987). Introducción a los métodos cualitativos de investigación: La búsqueda de significados. España: Paidós. Recuperado el 1 de junio de 2015, de: http://postgradofadecs.uncoma.edu.ar/archivos/loaizatsf/ Entrevista\%20Taylor\%20y\%20Bogdan.pdf.

Torcomian, C. (2015). Descripciones y relatos sobre la experiencia de llegada a la Universidad. Academicus. Revista de Ciencias de la Educación, 6, 25-39. Recuperado el 5 de julio de 2016, de: http://www.ice.uabjo.mx/images/academicus/Numero_6/Art3.pdf. 